\title{
Algorithms for identification and categorization
}

\author{
J.M. Cortes *, P.L. Garrido *, H.J. Kappen ${ }^{\dagger}$, J. Marro*, C. Morillas**, D. \\ Navidad* and J.J. Torres* \\ *Institute Carlos I for Theoretical and Computational Physics and Departamento de \\ Electromagnetismo y Física de la Materia, University of Granada, 18071-Granada, Spain \\ ${ }^{\dagger}$ Department of Biophysics, Radboud University of Nijmegen, 6525 EZ Nijmegen, The Netherlands \\ ** Departamento de Arquitectura y Tecnología de Computadores, University of Granada, \\ 18071-Granada, Spain
}

This talk reports on a series of efforts during the last decade aimed at modeling in a computer the cooperative properties that, according to some experimental evidence, could be relevant for the processing of patterns in a brain. In particular, a main recent interest is in designing fast and reliable algorithms for "restoring" a pattern, namely, identifying the original from which a highly degraded pattern proceeds. The challenge is to go beyond familiar methods, including Hopfield-like neural networks $[1,2]$ which, in spite of a great theoretical interest, are hampered in practice by the occurrence of mixture states, slow processing, limited capacity, and inadmissible statistical errors. Furthermore, we are concerned with an extended definition of "pattern", namely, we plan to deal from a high-resolution color picture to a complex pattern of behavior or the large set of qualities which serve to accurately identify a complex company, for example. This is relevant, in particular, to sociology, the focus in this meeting. However, the discussion below will avoid any specific application - except, eventually, for illustrative purposes.

Compared to machines, the human brain performs impressively in regards to our recent interest. Unfortunately, the brain strategy in these processes is not yet well understood. Nevertheless, there are now some indications which we shall take here as the basic hypothesis to build a model. Firstly, that processing of patterns is the result of cooperation between many units. Next, that associative memory in neurobiological systems is the consequence of, say, an essentially stochastic dynamics. That is, taking along the description to well-defined grounds, dynamics induces not only tendency toeards pure attractors, i.e., fixed points such as the ones in the Hopfield model, but also stochastic jumps between the attractos. Even more, the jumping is often restricted to the elements in one or a few of the classes in which one may assume the whole set of attractors to be partitioned. This possibility is interesting because it implies categorization, namely, classification of the stored patterns according to some criteria - e.g., the degree of correlation between the elements in the class(es). Such behavior has already been reported in several contexts, including the monkeys cortical activity and the insects early olfactory processing [3]-[7]. As a matter of fact, there is some evidence that recognition involves a previous process of class discrimination —indeed one may recognize a person as familiar and not being able to recall its identity [8].

The origin for this essential stochasticity in the dynamics of actual systems is likely

\author{
CP779, Modeling Cooperative Behavior in the Social Sciences: Eighth Granada Lectures \\ edited by P. L. Garrido, J. Marro, and M. A. Muñoz \\ (C) 2005 American Institute of Physics 0-7354-0266-3/05/\$22.50
}


to be at the synapses, which in practice determine much of the complex processing of information in the brain [9]. That is, the neural activity, which is intrinsically stochastic, further requires the continuous competition of (fast) "synaptic noise", which in turn is activity dependent, to accomplish the efficient transmission of information and a variety of computations [10,11]. Consequently, pursuing recent efforts [12], we found sensible investigating the effect of stochastic synapses on the fixed points of the retrieval processes in appropriate attractor neural networks. With this aim, we believed it convenient to model the synaptic noise based on the observation that synapses endure short-time depression. That is, periods of elevated presynaptic activity decrease the neurotransmitter release and, consequently, the postsynaptic response is depressed, which is likely to positively influence the transmission of information [12]-[15],[9].

I shall briefly illustrate here the above ideas in a simple model system which generalizes several proposals. Consider a set of units (neurons, social agents, etc.) that change randomly of state with time. Their randomness is regulated by a "temperature" parameter, $T_{0}$. Each unit is connected to each other with links, synapses, etc. whose intensities or weights change with time, also at random but regulated by a different "temperature", $T_{1}$. This sort of competition was first considered in Refs.[16]-[21]. To be specific, let $N$ binary (say) neurons with configurations $\mathbf{S}=\left\{s_{i}= \pm 1 ; i=1, \ldots, N\right\}$ connected by (say) synapses of weight $w_{i j} \in \Re ; i, j=1, \ldots, N$. The probability of the state $\Omega=(\mathbf{S}, \mathbf{W})$, with $\mathbf{W}=\left\{w_{i j}\right\}$, at time $t$ satisfies the familiar master equation, i.e., $\partial_{t} P(\boldsymbol{\Omega})=\sum_{\boldsymbol{\Omega}^{\prime}}\left[c\left(\boldsymbol{\Omega}^{\prime} \rightarrow \boldsymbol{\Omega}\right) P_{t}\left(\boldsymbol{\Omega}^{\prime}\right)-c\left(\boldsymbol{\Omega} \rightarrow \boldsymbol{\Omega}^{\prime}\right) P_{t}(\boldsymbol{\Omega})\right]$, with a transition rate which describes the indicated competition:

$$
c\left(\boldsymbol{\Omega} \rightarrow \boldsymbol{\Omega}^{\prime}\right)=p c^{\mathbf{W}}\left(\mathbf{S} \rightarrow \mathbf{S}^{\prime}\right) \delta(\mathbf{W}-\mathbf{W})+(1-p) c^{\mathbf{S}}\left(\mathbf{W} \rightarrow \mathbf{W}^{\prime}\right) \delta\left(\mathbf{S}-\mathbf{S}^{\prime}\right) .
$$

The elementary rates $c^{\mathbf{W}}$ and $c^{\mathbf{S}}$ are typically chosen by looking for simplicity [21]. For instance, one may take the former as a factorizable function of $2\left(s_{i} / T_{0}\right) h_{i}^{\mathbf{W}}(\mathbf{S})$, where $h_{i}^{\mathbf{W}}(\mathbf{S})=\sum_{j \neq i} w_{i j} s_{j}$ is the net (pre)synaptic current arriving to - or local field acting on- the (postsynaptic) neuron $i$. Here, $w_{i j}$ is determined in a previous learning process, e.g., by the Hebb's rule [22] from $M$ patterns which in this way remain stored in the system. It is to be remarked that, due to the generality we call for here, these patterns should be as diverse as to include from a set of orthogonal or near orthogonal patterns to a set of patterns that are highly correlated to each other.

A particularly interesting case occurs for $p \rightarrow 0$. In fact, this mimics rapid fluctuations in accordance with the observation of fast noise mentioned above. Furthermore, the model can then be solved analytically for some choices of parameters. One is lead in this limit, after rescaling time $t p \rightarrow t$, to a situation in which the neurons evolve as in the presence of a steady distribution for the synaptic fluctuations. The transition between states is then governed by an effective rate that involves the noise (effective) distribution. A principal result is that the ensuing stationary states are out of equilibrium [21]. This is because of the competition between the neural activity and the synaptic noise, which impedes reaching asymptotically equilibrium. This was first studied in some detail in Refs.[17, 18], and analysis of the intriguing behavior this may show concerning recognition was first reported in Refs.[19, 20].

Also remarkable is that, under appropriate conditions, which include parallel updating - as it is usual in cellular automata-, this system exhibits various retrieval phases. This 
is illustrated in Fig.1. In addition to a disordered phase (P) and a phase with associative memory $(\mathrm{F})$, there are regions in which one observes a continuous stochastic jumping between attractors $(\mathrm{O})$. Depending on the parameter values, this jumping may be rather complex [23]; see the right graph in Fig. 1. On the other hand, there is no region in the parameter space with mixture states such as the ones that hamper recognition in the Hopfield case [2, 19]. Also noticeable is that phase transitions may be of first-order, which implies that the recognition process may occur with negligible error [20].
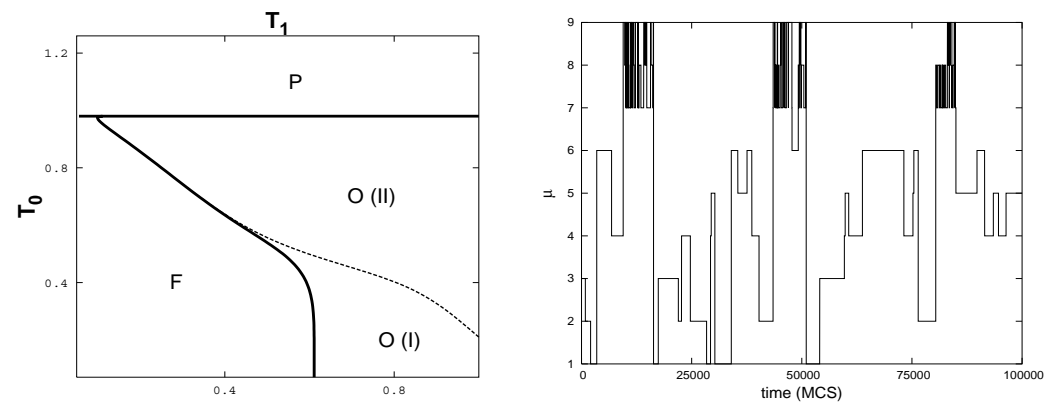

FIGURE 1. Left: Parameter space for 16384 neurons and 3 stored, highly-correlated patterns showing $\mathrm{P}$ and $\mathrm{F}$ phases, as defined in the text, and regions in which the state keeps jumping between patterns. The jumps are either uncorrelated or time-correlated for regions $\mathrm{O}(\mathrm{I})$ and $\mathrm{O}(\mathrm{II})$, respectively. Right: This shows, as a function of time, the order $\mu$ of the pattern which more closely resembles the current state of a network with parameters in region $\mathrm{O}(\mathrm{II})$. This is for $M=9$ correlated patterns stored in a set of $10^{4}$ fully connected neurons with $T_{0}=0.05$ and $T_{1}=1.25$ times the corresponding critical values. This shows how the neural automata constantly jumps between the stored patterns. The jumping is apparently random, but the system detects (upper part of the graph) a family — consisting of patterns 7, 8 and 9- whose elements are more correlated to each other than with the rest.

The same model was recently studied to show that the network topology importantly matters. It is unlikely that natural evolution leads to fully connected networks, and it was shown [24] that, in general, the capacity to store and retrieve is higher for a scale-free topology than for a comparable highly random-diluted network.

One may explicitly show that some of the jumping phenomena in the model - e.g., the one shown in the right graph of Fig. 1- is close to the reported "family discrimination" [8] by tuning the model details. For example, let us assume that the synaptic intensities are now $w_{i j}=\bar{w}_{i j} x_{j}$, where $\bar{w}_{i j}$ is fixed in a previous learning, as before, and $x_{j}$ is a stochastic variable. Let us assume that, in the limit $p \rightarrow 0$, the steady noise effective distribution is a product of functions $P\left(x_{j} \mid \mathbf{S}\right)=\zeta(\overrightarrow{\mathbf{m}}) \delta\left(x_{j}+\Phi\right)+[1-\zeta(\overrightarrow{\mathbf{m}})] \delta\left(x_{j}-\right.$ $1)$. Here, $\overrightarrow{\mathbf{m}}=\overrightarrow{\mathbf{m}}(\mathbf{S})$ is the $M$-dimensional vector of overlaps $m^{\nu}(\mathbf{S}) \equiv \frac{1}{N} \sum_{i} s_{i} \xi_{i}^{\nu}$, where $\xi^{\nu}=\left\{\xi_{i}^{\nu}= \pm 1, i=1, \ldots, N\right\}$ are the $M$ stored patterns, and $\zeta(\overrightarrow{\mathbf{m}})$ stands for a function of $\overrightarrow{\mathbf{m}}$ to be determined. This choice aims at modeling the reported short-term synaptic depression $[13,14]$. That is, increasing the mean firing rate results in decreasing the synaptic weight. The depression effect here depends on the overlap vector which measures the net current arriving to postsynaptic neurons.

The parameter space, namely, $(T, \Phi)$, where $\Phi$ is the parameter that controls now the noise intensity, also depicts the $P$ and $F$ phases, even for the case of sequential 

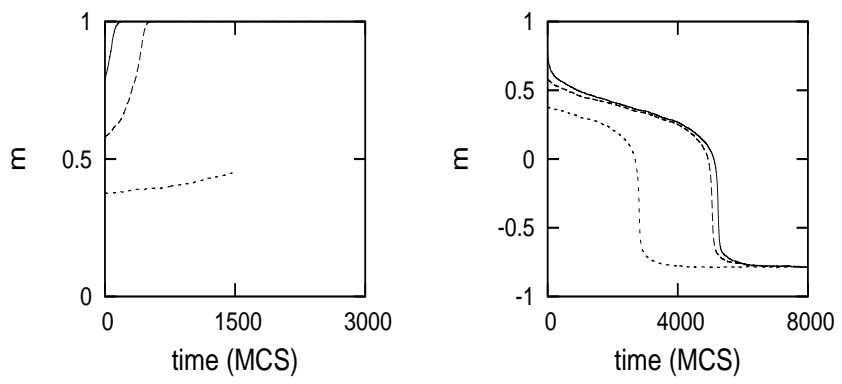

FIGURE 2. Time evolution of the overlap, as defined in the main text, between the current state and the stored pattern in Monte Carlo simulations with 3600 neurons at $T=0.1$. Each graph shows different curves corresponding to evolutions starting with different initial states. The two graphs are for $\delta=0.3$ and $\Phi=1$ (right) and $\Phi=-1$ (left), the former corresponding to the Hopfield case lacking the fast noise. This shows the important effect noise has on the network sensitivity to external stimuli. The same behavior occurs as one varies the external stimulation $\delta$.

updating [25]. The corresponding transition results of second order only for $\Phi>-4 / 3$, which includes the Hopfield classical limit $\Phi=-1$ (lack of depression). Otherwise, the transition is of first-order - with excellent recognition memory due to the sharp behavior just below the transition temperature. More specifically, there is a tricritical point is at $\left(T_{c}, \Phi_{c}\right)=(1,-4 / 3)$.

Also interesting are the consequences of the activity-dependent processes — which mimic short-term synaptic depression in the model - on the retrieval dynamics under external stimulation. That is, one may check the resulting sensitivity of the network to external inputs. A high degree of sensibility will facilitate the response to changing stimuli. In fact, this is an important feature of neurobiological systems which continuously adapt, and may thus quickly respond to varying stimuli from the environment. A simple external input may be simulated by adding to each local field a driving term $-\delta \xi_{i}, \forall i$, with $0<\delta \ll 1$ [12]. For a single pattern, $M=1$, this tries to move the network activity from the attractor, $\xi$, to the "antipattern", $-\xi$. The resulting behavior is illustrated in Fig. 2 [25].

The graphs in this figure, and similar evidence one may obtain for other parameter values, clearly demonstrate that presynaptic noise enhances the network sensitivity to a simple external stimulus. Furthermore, the jumping phenomena is robust with respect to the type of pattern stored. This is illustrated by comparison of the situation in Fig. 3 for uncorrelated patterns, i.e., patterns with mutual overlaps $m^{\nu, \mu} \equiv 1 / N \sum_{i} \xi_{i}^{\nu} \xi_{i}^{\mu} \approx 0$, and the one in Ref.[25] for correlated patterns, namely, $m^{\nu, \mu}=1 / 3$ for any two of them. On the contrary, there is no visible structure in the signal response in the absence of fast noise, e.g., in the Hopfield case as far as $\delta \ll 1$. As a matter of fact, the depth of the basins of attraction are large enough in the Hopfield model, to prevent any jumping phenomena for small $\delta$, except when approaching a critical point $\left(T_{c}=1\right)$, 


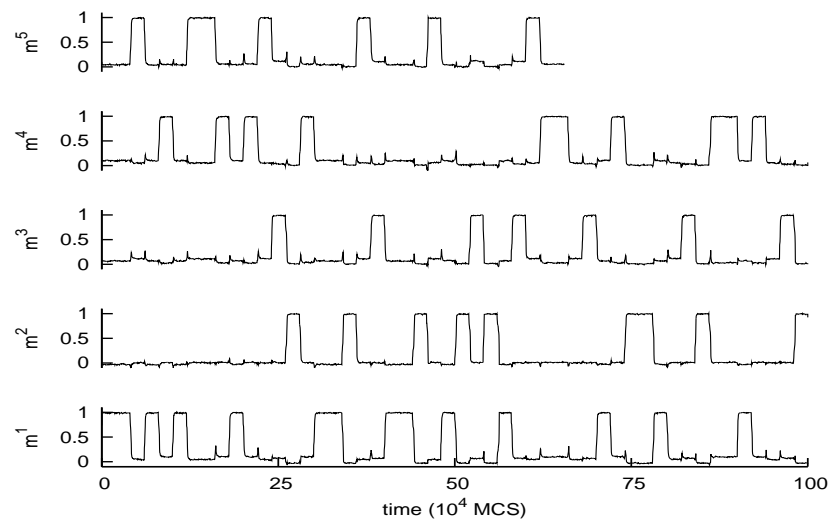

FIGURE 3. Time evolution of every overlap during a Monte Carlo simulation with 400 neurons, $M=5$ completely uncorrelated patterns, $\Phi=0.05$, and $T=0.1$. The system in this case was perturbed by the stimulus $-\delta \xi^{\nu}, \delta=0.3$, with the pattern index $\nu$ changed at random every 20,000 MC steps. The same experiment with $\Phi=-1$; i.e., the Hopfield case, produces a constant signal instead.

where fluctuations diverge. That is, adding fast noise in general seems to destabilize the fixed point for the interesting case of small $\delta$ far from criticality.

Summing up, the "two-temperatures model" [21] may naturally be adapted to incorporate certain neurobiological observations to obtain an interesting algorithm for memory recognition, including family discrimination. Using the language of neural networks, the model has two parameters, say, "temperatures" which control the stochastic dynamics of neurons and synaptic weights, respectively. We adapted this to study the consequences of fast synaptic noise on the attractor of a system with a finite number of stored patterns. Among the cases already considered, the most intriguing behavior ensues when the noise depends on the total presynaptic current arriving to the postsynaptic neuron. This case has been studied both numerically and analytically by using a mean-field hypothesis. The numerical work consists of a series of Monte Carlo simulations using both sequential Glauber, spin-flip dynamics and cellular automaton or Little (parallel updating) dynamics. Most available results concern the limit $\alpha=M / N \rightarrow 0$ but preliminary Monte Carlo simulations indicate that our results also hold for a macroscopic number of stored patterns, $\alpha \neq 0$.

A principal conclusion is that fast presynaptic noise may induce a nonequilibrium condition which results in an important intensification of the network sensitivity to external stimulation. One explicitly sees that the noise may turn unstable the attractor or fixed point solution of the retrieval process, and the system then seeks for another attractor. In particular, one observes jumping from the stored pattern to the corresponding antipattern for $M=1$, and jumping between patterns for a larger number of stored patterns. This behavior is most interesting because the network ability to detect changing stimuli from the environment is thus improved. We observe the jumping to be very sensitive to the forcing stimulus, but rather independent of the network initial state or the 

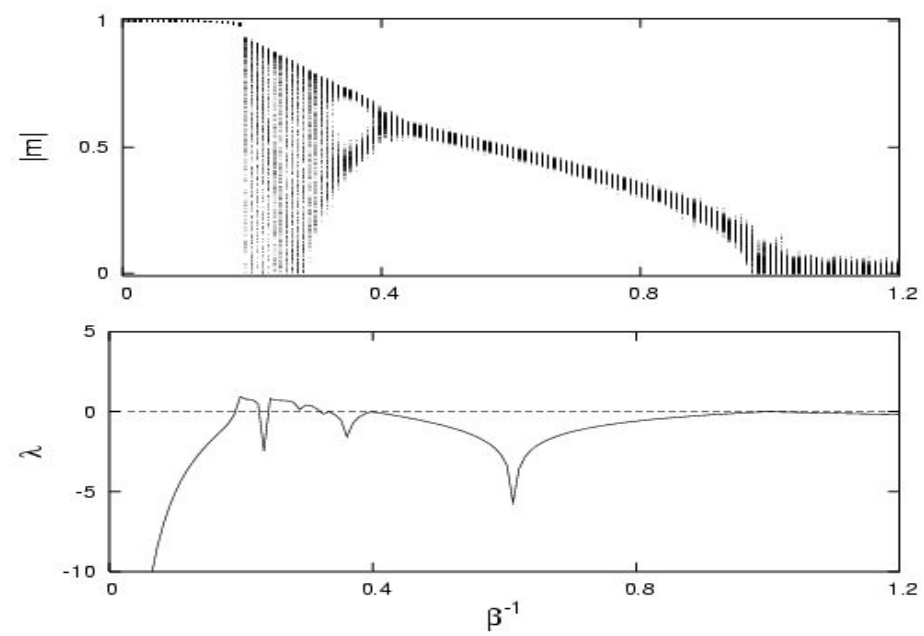

FIGURE 4. The top graph shows, for $M=1$, the absolute value of the overlap as a function of the (neuron) temperature for $\Phi=0.05$. This illustrates, in a MC simulation with $10^{4}$ neurons, irregular behavior and a transition at $T_{C}=1$ from the $\mathrm{F}$ phase to the $\mathrm{P}$ phase as the temperature is increased. The bottom graph shows the corresponding Lyapunov exponent, as obtained from an accurate mean-field description. This illustrates eventual chaotic behavior, i.e., $\lambda>0$.

thermal noise. We also observe that the jumping may become chaotic when the system is implemented as a cellular automaton with parallel updating. This is demonstrated by our mean-field computation of the corresponding Lyapunov exponent which is depicted in Fig. 4.

Finally, one may argue that, besides recognition, the processes of class identification and categorization in nature might follow our model strategy. That is, different attractors may correspond to different objects, and a dynamics conveniently perturbed by fast noise may keep visiting the attractors belonging to a class which is characterized by a certain degree of correlation between its elements. In fact, as described above, it was recently reported that a similar mechanism seems at the basis of several natural phenomena.

We acknowledge financial support from MCyT-FEDER (project No. BFM2001-2841 and Ramón y Cajal contract) and MADOC.

\section{REFERENCES}

1. J.J. Hopfield, Proc. Natl. Acad. Sci. USA 79, 2554(1982)

2. D. Amit, H. Gutfreund, and H. Sompolinsky, Ann. Phys. 173, 30 (1987)

3. M. Abeles, H. Bergman, I. Gat, I. Meilijson, E. Seidelman, N. Tishby, and E. Vaadia, Proc. Natl. Acad. Sci. USA 92, 8616 (1995) 
4. L.M. Miller and C.E. Schreiner, J. Neurosci. 20, 7011 (2000)

5. S. Scarpetta, L. Zhaopin, and J. Hertz, Neural Comp. 14, 2371 (2002)

6. T. Oyamada, Y. Kashimori, O. Hoshino, and T. Kambara, Biological Cybernetics 83, 21 (2000)

7. G. Laurent, M. Stopfer, R. Friedrich, M. Rabinovich, A. Volkovskii, and H. Abarbanel, Annu. Rev. Neurosci. 24, 263 (2001)

8. R. Bogacz and M.W. Brown, Hippocampus 13, 494 (2003)

9. L.F. Abbott and W.G. Regehr, Nature 431, 796 (2004)

10. C. Allen and C. Stevens, Proc. Nat. Acad. Sci. USA 91, 10380 (1994)

11. A. Zador, J. Neurophysiology 79, 1219 (1998)

12. D. Bibitchkov, J.M. Herrmann, and T. Geisel, Network: Comput. Neural Syst. 13, 115 (2002)

13. M. Tsodyks, K. Pawelzik, and H. Markram, Neural Comp. 10, 821 (1998)

14. L. Pantic, J.J. Torres, and H.J. Kappen, Neural Comp. 14, 2903 (2002)

15. A.M. Thomson, A.P. Bannister, A. Mercer, and O.T. Morris, Phil. Trans. R. Soc. London B 357, 1781 (2002)

16. P.L. Garrido and J. Marro, J. Phys. A: Math. Gen. 25, 1453 (1992)

17. P.L. Garrido and J. Marro, Lecture Notes in Computer Science (Springer-Verlag) 540, 25 (1992)

18. J.J. Torres, P.L. Garrido, and J. Marro, J. Phys. A: Math. Gen. 30, 7801 (1997)

19. J. Marro, P.L. Garrido, and J.J. Torres, Phys. Rev. Lett. 81, 2827 (1998)

20. J. Marro, J.J. Torres, and P.L. Garrido, J. Stat. Phys. 94, 837 (1999)

21. J. Marro and R. Dickman, Nonequilibrium Phase Transitions in Lattice Models, Cambridge Univ. Press, Cambridge 1999.

22. D.O. Hebb, The Organization of Behavior, Wiley, New York 1949.

23. J.M. Cortes, P.L. Garrido, J. Marro, and J.J. Torres, Neurocomputing 58-60, 67 (2004)

24. J.J. Torres, M.A. Muñoz, J. Marro, and P.L. Garrido, Neurocomputing 58-60, 229 (2004)

25. J.M. Cortes, J.J. Torres, J. Marro, P.L. Garrido, and R. Kappen, "Effects of Fast Presynaptic Noise in Attractor Neural Networks", preprint.

26. J.M. Cortes et al, to be published. 